\title{
Nucleosynthesis in extremely metal-poor intermediate-mass stars
}

\author{
Monique A. Cruz* \\ Universidade de São Paulo \\ E-mail: macruz@usp.br \\ Silvia Rossi \\ Universidade de São Paulo \\ E-mail: silvia.rossieiag.usp.br
}

We have performed evolutionary and nucleosynthesis calculations of extremely metal-poor intermediate-mass stars from the ZAMS to the TP-AGB. Our models undergo the so called proton ingestion episode (PIE). This episode occurs in the first(s) thermal pulse(s) for extremely metal-poor stars with masses $\mathrm{M}>1.0 \mathrm{M}_{\odot}$. As expected, our models are highly enriched in $\mathrm{CNO}$ elements after the PIE. Lithium is also produced in our models with abundances varying from $\log \varepsilon(\mathrm{Li})=1.59$ to 3.29 . In addition, neutrons are produced during the PIE in our models, given rise to significant s-process surface enhancement in some models. Moreover, europium can be also produced in a significant amount $([\mathrm{Eu} / \mathrm{Fe}] \sim 2.0)$ already in the first TP.

XIII Nuclei in the Cosmos

7-11 July, 2014

Debrecen, Hungary

\footnotetext{
*Speaker.
} 


\section{Introduction}

The metallicity of a star is an indicator of the degree of pollution its gas has been subject to since the Big Bang. The lower the metallicity the lower the number of stellar generations that polluted the interstellar medium. Extremely metal-poor stars, hereafter EMP stars, have less then $1 / 1000$ th of the iron content of the sun $([\mathrm{Fe} / \mathrm{H}]<-3.0,[1])$ and might provide important clues about individual nucleosynthesis processes that happened in the early stages of our Galaxy. Over the course of the past decades these stars have been studied with increasing accuracy. While approximately $10 \%$ of metal-poor stars are enhanced in carbon, for the EMP stars this number increases to about $30 \%$.

These carbon-enhanced metal-poor stars, hereafter CEMP stars, present a diversity of abundance patterns and are usually divided into four subclasses: CEMP-s ( $[\mathrm{Ba} / \mathrm{Fe}]>+1.0$ and $[\mathrm{Ba} / \mathrm{Eu}]>+0.5)$, CEMP-r/s $\quad([\mathrm{Ba} / \mathrm{Fe}]>+1.0$ and $0.0<[\mathrm{Ba} / \mathrm{Eu}]<+0.5), \quad$ CEMP-r $([\mathrm{Eu} / \mathrm{Fe}]>+0.3$ and $[\mathrm{Ba} / \mathrm{Eu}]<0.0)$, and CEMP-no $([\mathrm{Ba} / \mathrm{Fe}]<0.0)$. The s-process and carbon enhancements in CEMP-s stars are believed to have been originated from a former AGB companion that dumped enriched material onto the still visible star. It has been found that a large fraction of CEMP-s stars are in binary systems [2] which corroborates this scenario. On the other hand, the origin of the s- and r-process enhancements in CEMP-r/s stars is still open to debate. While some studies have claimed that the $\mathrm{Ba}$ and Eu present in these stars should be produced in different astrophysical sites, the tight correlation between $[\mathrm{Ba} / \mathrm{Fe}]$ and $[\mathrm{Eu} / \mathrm{Fe}]$ found by the observations for both CEMP-s and CEMP-r/s indicates that these two elements might have the same origin [3].

EMP stars might undergo proton ingestion episode, hereafter PIE, during the core-helium flash $\left(\mathrm{M}<1.2 \mathrm{M}_{\odot}\right)$ or the TP-AGB phase $\left(1.2 \mathrm{M}_{\odot}<\mathrm{M}<3.0 \mathrm{M}_{\odot}\right)$. The ignition of He-burning results in the formation of the so-called helium convective zone (HeCZ). The outer boundary of the $\mathrm{HeCZ}$ advances in mass during the development of the He-flash. In the metal-poor environment, the entropy barrier between the He- and H-burning shells is very low. This results in a penetration of the $\mathrm{HeCZ}$ into $\mathrm{H}$-rich layers [4]. Once the $\mathrm{HeCZ}$ reaches the $\mathrm{H}$-rich shell, protons are mixed down into the convective zone and captured by the abundant ${ }^{12} \mathrm{C}$. The burning timescale for hydrogen through the reaction ${ }^{12} \mathrm{C}(\mathrm{p}, \gamma)$ becomes shorter as protons travel through the $\mathrm{HeCZ}$. There is a point inside the $\mathrm{HeCZ}$ where the mixing and burning timescales are comparable. At this position the energy released by H-burning is maximum and will lead to a secondary flash, the H-flash. At the position of the H-flash, the $\mathrm{HeCZ}$ splitts into two convective zones. The upper convetive zone will advance outward until the envelope deepens down and products of the nucleosynthesis during and after the PIE are transported to the surface.

Carbon, nitrogen, and oxygen are the main products of the PIE which are trasported to the surface. Lithium can also be produced during the PIE [5-8]. In addition, the ${ }^{13} \mathrm{C}$ produced during the PIE might capture an alpha particle and produce neutrons through the reaction ${ }^{13} \mathrm{C}(\alpha, \mathrm{n}){ }^{16} \mathrm{O}$. The amount of neutrons released in the PIE is larger than what is found in the normal TP-AGB phase $\left(\mathrm{N}_{\mathrm{n}}>10^{10} \mathrm{~cm}^{-3}\right)$ and might lead to a large production of s-process elements [7, 8]. Moreover, this large neutron flux might also lead to the production of large amounts of europium. Therefore, the PIE might be an important production site for the s-process and r-process elements found in the CEMP-s/r subclass.

We present here preliminary analysis of nucleosynthesis calculations of intermediate-mass 
EMP stars. A detailed discussion will be presented in a forthcoming paper.

\section{Models and Results}

We have performed evolutionary and nucleosynthesis calculations from the zero-age main sequence to the TP-AGB phase using the GARSTEC evolutionary code as described in [8]. The models have masses $\mathrm{M}=2.0$ and $3.0 \mathrm{M}_{\odot}$ and metallicities $\mathrm{Z}=10^{-8}, 10^{-7}$, and $10^{-5}$. The composion is solar-scaled in all models. Convection is modeled using the Mixing Length Theory and the mixing length parameter $\alpha=1.75$. Our models include overshooting in all convective boundaries with the standard choice of free parameter $\mathrm{f}=0.016$ (More details in [8]). [8] have discussed the effect of overshooting in the PIE and the s-process production for low-mass EMP stars. They have shown that a more vigorous entrainment of hydrogen into the $\mathrm{HeCZ}$ leads to a smaller final neutron exposure, favoring the production of lighter s-process elements. We expect similar results for the intermediate-mass case if a larger value of $\mathrm{f}$ is used.

PIE occurs in the first thermal pulse in all models. Its duration $\left(\Delta\right.$ tPIE $\left._{\text {II }}\right)$ varies from $\sim 10^{-3} \mathrm{yr}$ to $\sim 5 \times 10^{-1} \mathrm{yr}$, depending on mass and metallicity. While in the $2.0 \mathrm{M}_{\odot}$ models the duration of the PIE increases with increasing metallicity, in the $3.0 \mathrm{M}_{\odot}$ no clear trend with metallcity has been found. All models are highly enhanced in CNO elements after the post-PIE dredge-up. However, whilst PIE in low-mass EMP stars increases the $\mathrm{CNO}$ abundance to values larger than $\mathrm{Z}_{\mathrm{CNO}}=10^{-2}$ [8], our intermediate-mass models present $\mathrm{CNO}$ surface abundances ranging between $10^{-4}$ and $10^{-3}$.

The time between the H-flash and the start of the post-PIE dredge-up $\left(\Delta \mathrm{t}_{\mathrm{d}}\right)$ is an important quantity affecting the lithium production. If $\Delta \mathrm{t}_{\mathrm{d}}$ is long enough ${ }^{7} \mathrm{Li}$ is converted into ${ }^{4} \mathrm{He}$ through proton capture reactions before it is transported to the surface. In our models, $\Delta \mathrm{t}_{\mathrm{d}}$ varies from 0.20 to $\sim 15 \mathrm{yr}$. The $3.0 \mathrm{M}_{\odot}$ model with metallicity $\mathrm{Z}=10^{-5}$ which presents the largest $\Delta \mathrm{t}_{\mathrm{d}}$, has the lowest lithium surface abundance $(\log \varepsilon(\mathrm{Li})=1.59)$. On the other hand, the largest lithium abundance $(\log \varepsilon(\mathrm{Li})=3.29)$ is shown by the $2.0 \mathrm{M}_{\odot}$ model with metallicity $\mathrm{Z}=10^{-5}$. Although this model does not have the smallest $\Delta \mathrm{t}_{\mathrm{d}}$, it has the deepest $\mathrm{H}$-flash position, implying in a larger temperature at the bottom of the upper convective shell after the splitting. A larger temperature leads to a larger reaction rate for the reaction ${ }^{3} \mathrm{He}(\alpha, \gamma){ }^{7} \mathrm{Be}$ and therefore, a larger lithium production. The other light elements (F, Na, Mg, Al) do not show a clear trend with neither mass, metallicity or any PIE property (Figure 1).

The proton ingestion episode is an important site for neutron production $[5-8]$. The ${ }^{13} \mathrm{C}$ produced by the reaction ${ }^{12} \mathrm{C}(\mathrm{p}, \gamma)$ captures the abundant ${ }^{4} \mathrm{He}$ in the $\mathrm{HeCZ}$, producing neutrons. Our models achieve neutrons fluxes larger than $\mathrm{N}_{\mathrm{n}}=10^{10} \mathrm{n} / \mathrm{cm}^{-3}$ during the PIE. ${ }^{14} \mathrm{~N}$ is an important neutron poison. If more ${ }^{14} \mathrm{~N}$ is present in the environment than ${ }^{13} \mathrm{C}$ (hence, ${ }^{13} \mathrm{C} /{ }^{14} \mathrm{~N}<1.0$ ), neutrons are captured by nitrogen through the reaction ${ }^{14} \mathrm{~N}(n, p){ }^{14} \mathrm{C}$, instead of being used for the s-process nucleosynthesis. In our models the ${ }^{13} \mathrm{C} /{ }^{14} \mathrm{~N}$ ratio is larger than unity, favoring the production of s-process elements. Figure (1) shows the production of some selected heavy elements during the PIE. As shown by [8], the important quantity defining the final s-process surface abudance is the average neutron exposure in the convective zone. This quantity depends on the duration of the PIE and the size of the convective zone. Models with $2.0 \mathrm{M}_{\odot}$ and metallicities $\mathrm{Z} \leq 10^{-7}$, do not last long enough to produce elements beyond strontium. On the other hand, despite the longer 


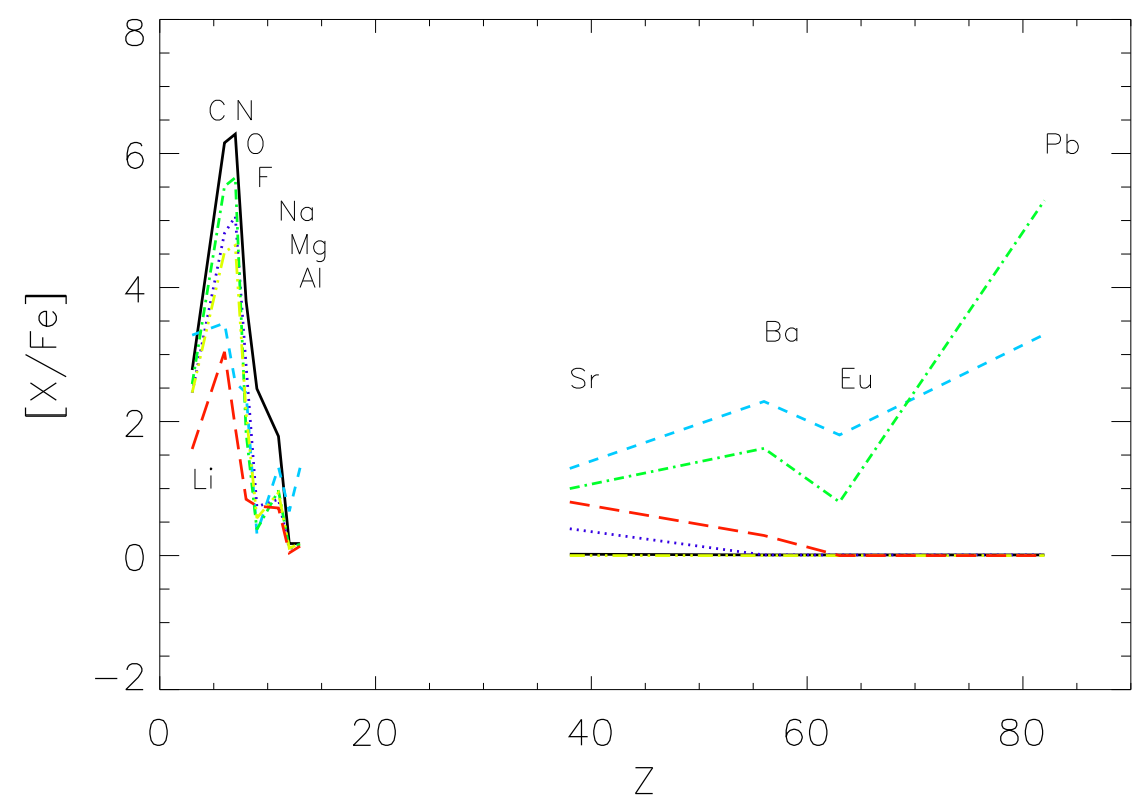

Figure 1: The surface abundance distribution for light elements $(\mathrm{A} \leq 27)$ and for some selected heavy elements after the PIE. Black solid line indicates model with $2.0 \mathrm{M}_{\odot}$ and $Z=10^{-8}$, light blue dashed line indicates model with $2.0 \mathrm{M}_{\odot}$ and $Z=10^{-7}$, dark blue dotted line indicates model with $2.0 \mathrm{M}_{\odot}$ and $Z=10^{-5}$, green dot-dashed line indicates model with $3.0 \mathrm{M}_{\odot}$ and $Z=10^{-8}$, yellow line indicates model with $3.0 \mathrm{M}_{\odot}$ and $Z=10^{-7}$, and red dashed line indicates model with $3.0 \mathrm{M}_{\odot}$ and $Z=10^{-5}$.

duration of the PIE $\left(\Delta \mathrm{t}_{\mathrm{PIE}} \sim 5 \times 10^{-1} \mathrm{yr}\right.$ ) in the $2.0 \mathrm{M}_{\odot}$ model with metallicity $\mathrm{Z}=10^{-5}$, the larger size of the $\mathrm{HeCZ}$ leads to a smaller production of $\mathrm{Pb}$ in comparison to the $3.0 \mathrm{M}_{\odot}$ model with metallicity $\mathrm{Z}=10^{-8}\left(\Delta \mathrm{t}_{\mathrm{PIE}} \sim 2 \times 10^{-1} \mathrm{yr}\right)$.

We have found a variety of behaviours for the $[\mathrm{Ba} / \mathrm{Eu}]$ ratio in our models. While some models present the usual $[\mathrm{Ba} / \mathrm{Eu}]>0.5$ found in AGB models and compatible with CEMP-s observations, the $2.0 \mathrm{M}_{\odot}$ model with metallicity $\mathrm{Z}=10^{-5}$ shows $[\mathrm{Ba} / \mathrm{Eu}]=0.5$ and $[\mathrm{Eu} / \mathrm{Fe}] \sim 2.0$, compatible with the CEMP-r/s abundance values. This suggests that the PIE might be a potential site for the production of the CEMP-r/s subclass and further investigation is necessary.

Acknowledgements: M.A. Cruz wishes to acknowledge FAPESP financial support under the Fund 2013/16924-7. S. Rossi thanks the partial support from FAPESP, CNPq, and CAPES.

\section{References}

[1] T.C. Beers, N. Christlieb, ARA\&A 43 (2005) 531

[2] S. Lucatello, S. Tsangarides, T. C. Beers, E. Carreta, R. G. Gratton, S. G. Ryan, ApJ 625 (2005) 825

[3] D. M. Allen, S. G. Ryan, S. Rossi, T. C. Beers, S. A. Tsangarides, A\&A 548 (2012) A34

[4] D. Hollowell, I. Jr. Iben, M. Y. Fujimoto, ApJ 351 (1990) 245

[5] N. Iwamoto, T. Kajino, G. J. Mathews, M. Y. Fujimoto, W. Aoki, ApJ 602 (2004) 377 
[6] S. Cristallo, L. Piersanti, O. Straniero, R. Gallino, I. Dominguez, F. Kaeppeler, PASA 26 (2009) 139

[7] S. W. Campbell, M. Lugaro, A. I. Karakas, A\&A 522 (2010) L6

[8] M. A. Cruz, A. Serenelli, A. Weiss, A\&A 559 (2013) A4 